\title{
Erratum to: Discriminating Dabry's sturgeon (Acipenser dabryanus) and Chinese sturgeon (A. sinensis) based on DNA barcode and six nuclear markers
}

\author{
Jiuxuan Li • Dongqi Liu - Qingzhan Ma • \\ Xiuyue Zhang - Wei Dai - Yongbo Chen • \\ Yong Liu $\cdot$ Zhaobin Song
}

Published online: 28 September 2015

(C) Springer International Publishing Switzerland 2015

Due to an unfortunate turn of events, Fig. 2 appeared incorrectly in the original publication causing the

The online version of the original article can be found under doi:10.1007/s10750-015-2251-z.

J. Li · D. Liu $\cdot$ Q. Ma $\cdot$ X. Zhang $\cdot$ Z. Song $(\bowtie)$

Sichuan Key Laboratory of Conservation Biology on Endangered Wildlife, College of Life Sciences, Sichuan University, Chengdu 610065, People's Republic of China e-mail: zbsong@scu.edu.cn

\section{Zhang $\cdot$ Z. Song}

Key Laboratory of Bio-Resources and Eco-Environment of Ministry of Education, College of Life Sciences,

Sichuan University, Chengdu 610065,

People's Republic of China

caption to mismatch the figure. The correct image and its caption are published here and should be treated as definitive by the reader.

\section{W. Dai · Y. Chen}

China Three Gorges Corporation, Beijing 100038,

People's Republic of China

Y. Liu

Jinsha River Xiluodu Xiangjiaba Hydropower Station of

Rare and Unique Fish Multiplication Station,

Yibin 644612, People's Republic of China 


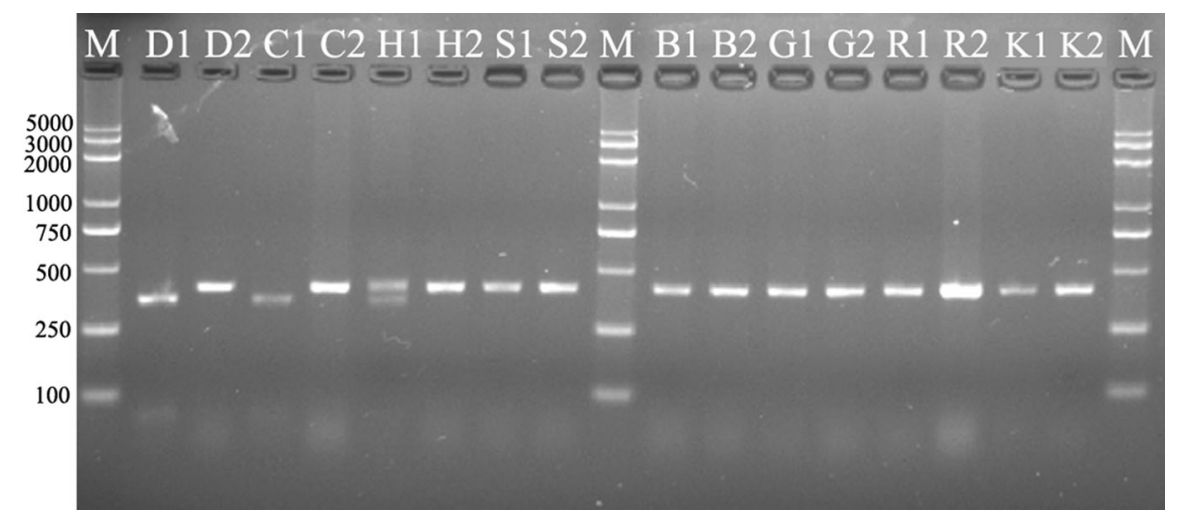

Fig. 2 Electrophoretogram of TYR gene fragments for different sturgeons, indicating the restriction enzyme cutting effects of the gene by Nco I. Dabry's sturgeon $(D)$, Chinese sturgeon $(C)$, hybrid Acipenser dabryanus-baerrii $(H)$, A. schrenckii $(S)$,
A. baerrii $(B)$, A. gueldenstaedtii $(G)$, A. ruthenus $(R), H$. dauricus $(K) ; 1$ after digestion, 2 before digestion by Nco I. $M$ Marker 